\title{
Innovative reflection on oral cancer research priorities: the contribution of social network analysis
}

\author{
Allana PIVOVAR(a) i \\ Elder SEMPREBON ${ }^{\text {(b) }}$ \\ Camila Pinheiro FURQUIM(c) \\ Juliana Lucena SCHUSSEL ${ }^{(a)}$ \\ José Miguel AMENÁBAR(a) \\ Cassius Carvalho TORRES-PEREIRA ${ }^{(a)}$ \\ (a) Universidade Federal do Paraná - UFPR, \\ School of Dentistry, Graduate Program in \\ Dentistry, Curitiba, PR, Brazil. \\ (b) Universidade Federal do Paraná - UFPR, \\ Graduate Program in Business, Curitiba, \\ PR, Brazil. \\ (c) Universidade de Guarulhos - UnG, \\ Dental Research Division, Department of \\ Periodontology and Oral Implantology, \\ Guarulhos, SP, Brazil.
}

\begin{abstract}
The objective of this study is to present a tool to help understand how variables associated with oral cancer prevention relate to each other in a social network. A search of the Scopus database was performed using terms related to oral cancer and prevention from 2000 to 2020. The keywords were used as nodes and were analyzed using NodeXL, which produced the network graphic analysis. From the 1004 publications available, 4038 different keywords were obtained and then grouped into 75 constructs based on conceptual similarity. The most influential nodes were risk factors, comorbidities, epidemiology, and treatment. However, topics such as technology, telemedicine, selfexamination, and diagnostic delay remain far removed from central relations. Network analysis enabled us to observe the bias of biological and basic science in the field and identify a need for studies concerning primary prevention, behavioral interventions, and inequalities in oral cancer.
\end{abstract}

Keywords: Mouth Neoplasms; Social Network Analysis.

\section{Introduction}

Declaration of Interests: The authors certify that they have no commercial or associative interest that represents a conflict of interest in connection with the manuscript.

Corresponding Author:

Allana Pivovar

E-mail: lana.pivovar@gmail.com

https://doi.org/10.1590/1807-3107BOR-2022.vol36.0023

Submitted: April 15, 2021

Accepted for publication: September 9, 2021

Last revision: October 26, 2021
Oral cancer, representing all malignancies that occur in the oral cavity, is the eighth-most incidental neoplasm worldwide. ${ }^{1}$ The etiologies of oral cancer include tobacco intake, smoking, alcohol and areca nut intake, excessive sunlight exposure, and human papilloma virus (HPV). ${ }^{2}$ Despite therapeutic improvements, the survival rate has not changed significantly over the past few decades, with a 5-year survival rate slightly above $50 \%{ }^{3}$

Oral cancer is particularly dangerous because it is relatively painless in its early stages. ${ }^{4}$ Consequently, it is usually discovered in its late stages and is therefore associated with poor prognosis. Treatment can be very expensive; therefore, preventive measures are of great importance. Prevention requires a multidisciplinary approach involving coordinated efforts from all sectors of society. ${ }^{2}$ Primary preventive measures should include an increase in public awareness of the risk factors and changes in individual's behavior; secondary preventive measures include regular oral head and neck examinations and treatment of potentially malignant conditions or in situ neoplasms. ${ }^{5}$ Consequently, tertiary prevention aims to prevent redevelopment. ${ }^{2}$

Network analysis facilitates a visual demonstration of the positions and relationships between the variables, with two fundamental elements: 
nodes and edges. In a graph, nodes are represented by circles, whereas edges are lines that connect these circles. Conceptually, nodes can represent many things, including people, organizations, websites, or keywords. The edges can represent connections in social networks, several relationships, hyperlinks, or relationships between variables, as in this study. Another significant contribution of this technique is the representation of the study of a topic through welldefined clusters. These clusters help clarify whether a particular subject has subtopics, divisions, or groups, allowing a detailed understanding of what is being researched. Statistical data allow quantitative analysis of the relevance of a topic (node) or its importance within a set (network) of related variables. ${ }^{67,8}$

Network analysis in science helps to identify influential terms based on several metrics. This is conceptually important because social influence has different definitions and measures of importance, called centralities ${ }^{9}$. These are degree centrality, closeness centrality, betweenness centrality, and eigenvector, which are described below. These basic centrality measures have been used extensively in the healthcare literature to quantify the network positions of individual actors in networks., ${ }^{9,1,11}$

The network analysis approach could represent an important contribution for scholars interested in oral cancer, as it identifies research gaps based on the strength of connections between keywords. The main goal of this study was to present a social network analysis of keywords used in studies in the field of oral cancer prevention.

\section{Methodology}

\section{Eligibility criteria and Information source}

To be included in this study, papers needed to present a minimum of one of the keywords identified by the authors and be published in English between January 2000 and April 2020.

\section{Search}

To identify potentially relevant documents, the Scopus database was examined, and the following terms were used: ("Oral cancer" OR "Oral squamous cell carcinoma" OR "mouth cancer" OR "oral malignancies" OR "oral neoplasm" OR "lip cancer" OR "head and neck cancer") AND (prevention OR "Preventive measures" OR preventive).

\section{Study selection}

The electronic search was performed independently by two authors (A.P. and C.P.F.). Information such as author, year, title, and keywords were organized in an Excel spreadsheet, and a third researcher (E.S.), experienced in network analysis, cleaned and excluded papers that did not include the author's keywords and all duplicates were removed. Author keywords were chosen because they were more available and abundant

\section{Data collection process}

To avoid an overload of information in the presentation of the network, the keywords were grouped manually into constructs based on nature and conceptual similarity. Initially, two oral medicine specialists (A.P and C.P.F) independently classified all keywords into constructs. Then, three experienced oral medicine specialists (C.C.T.P., J.M.A.C., and J.S.) reviewed all the keywords and suggested the main constructs by consensus. Inconsistencies in the classification were revised and solved by all researchers. Inter-observer agreement was not calculated. More details about the spreadsheet organization can be found in the supplemental material.

\section{Geometry of the network}

The data were analyzed using NodeXL with Microsoft Excel 2007/2010 version 1.0.1.361. The layout of the chosen graphic was a non-directional type named Fruchterman-Reingo, which was created automatically. It included network metrics such as centrality, centralization, density, distance, bonding, and bridging, to understand how the system was operating at each of the three points in time.

\section{Results}

\section{Study selection}

A total of 1495 studies were identified using the search strategy described above. Duplicates were 
removed, and 1004 papers with 4038 author keywords were included. All of these studies were published between 2000 and 2020. The selection process is shown in Figure 1.

\section{Summary of network geometry}

The proposed network contained 75 vertices and 372 unique relations (Figure 2). The nodes with larger measures of centralities are, naturally, those eligible as search terms (oral cancer, prevention, head and neck cancer, oral malignancies); therefore, we ignored them in the presentation of the results to better explore other interactions. The study design also had higher centrality measurements because we grouped all the different study methods into a single construct. Consequently, this will also be ignored in this textual data presentation. The full details of the data are shown in Table.

\section{Degree centrality}

The degree of centrality indicates the activity of the nodes and their popularity in a network.
A construct with a high degree of centrality in a social network has a high number of direct connections with other network actors. ${ }^{8}$ Our study is a measure of the most popular topics of the articles. The ten constructs that had the highest measures of degree centrality in the network were risk factors (64), comorbidities (63), epidemiology (59), other cancer topography (59), treatment (57), chemoradiotherapy (57), post-treatment complications (57), screening (56), molecular biology/biomarkers, and potentially malignant conditions (53).

\section{Closeness centrality}

Closeness centrality represents the reachability of an actor in relation to other actors in a network. Closeness can be regarded as a measure of how fast information can be spread to all other nodes. The ten constructs that had the highest values of closeness centrality in the network were risk factors (0.012), comorbidities (0.011), other cancer topographies (0.011), epidemiology (0.011), treatment (0.0108), chemoradiotherapy (0.0108), post-treatment

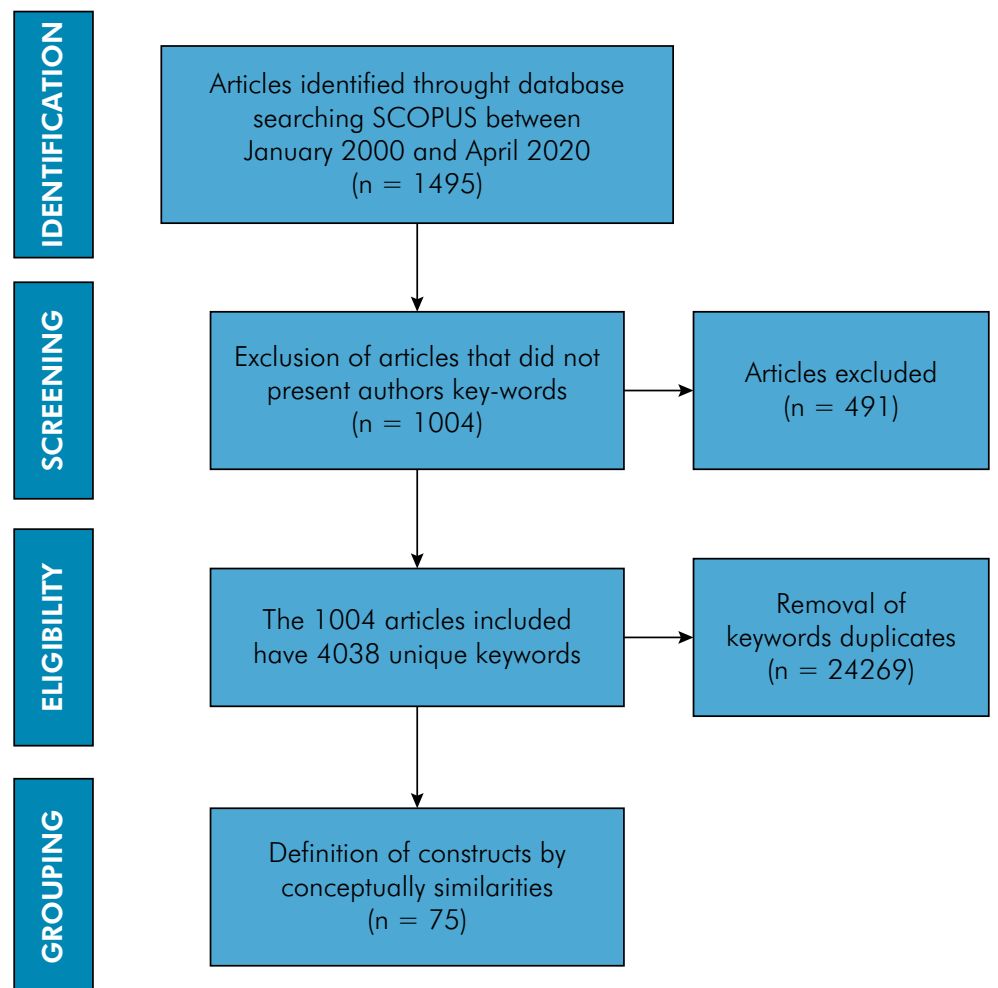

Figure 1. Flow chart of methodological steps. 


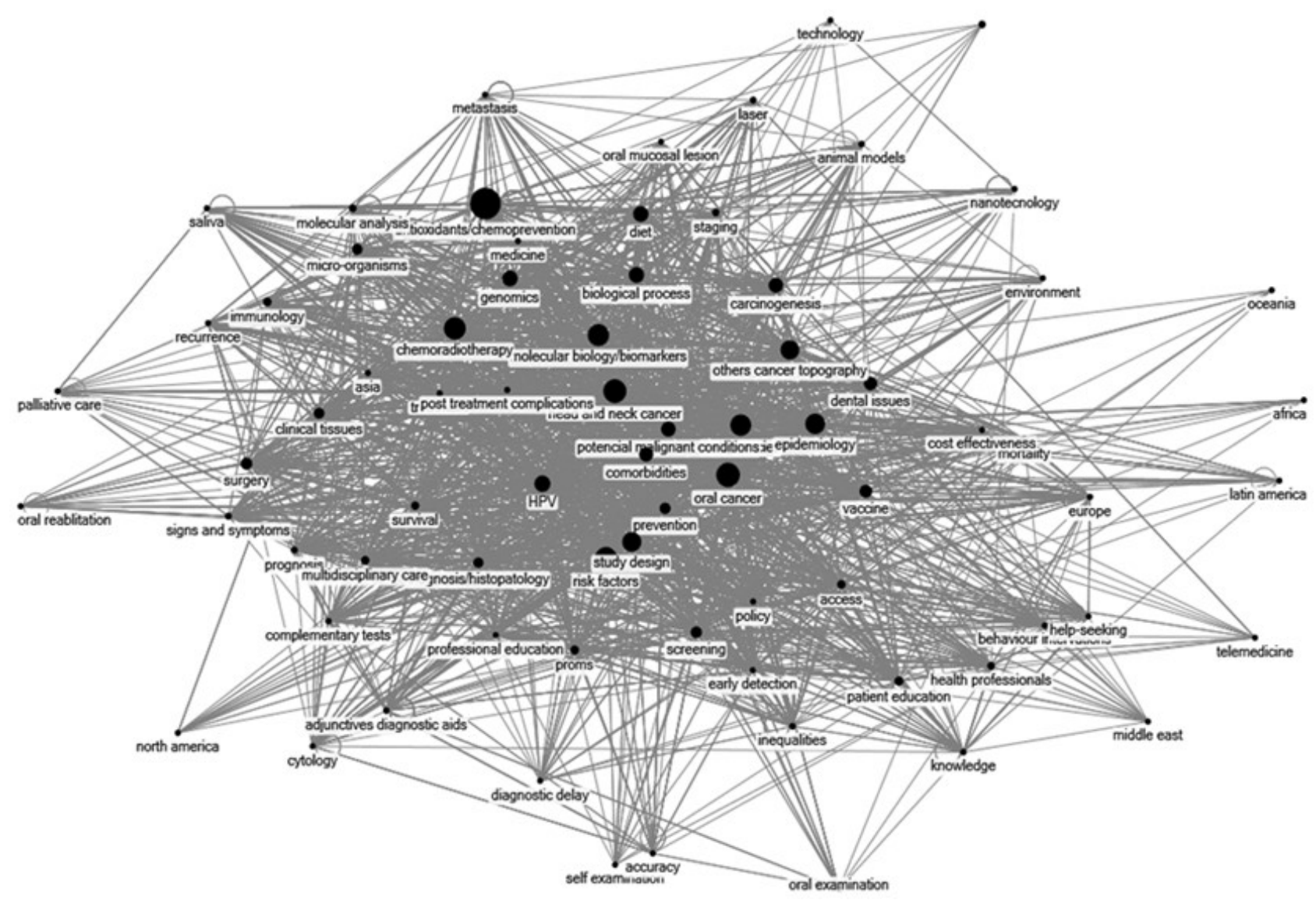

Figure 2. Network of variables on oral cancer.

complications (0.0108), screening (0.0106), molecular biology/biomarkers (0.0104), and potential malignant conditions (0.0103).

\section{Betweenness centrality}

The betweenness centrality represents the capacity of a node to control the flow of information in the network between any pair of nodes in the network. The underlying assumption of the betweenness centrality is that 'nodes in the middle' have more 'Intra organizational influence' on others in a network. In other words, it reflects the nodes that serve as bridges between the vertices of the network. The ten constructs that had the highest measures of betweenness centrality in the network were comorbidities (76.04), other cancer topography (63.96), risk factors (52.67), screening (48.33), post-treatment complications (40.29), epidemiology (38.38), chemoradiotherapy (35.35), early detection (29.36), carcinogenesis (27.59), and dental issues (26.95).

\section{Eigenvector}

The eigenvector is an ideal measurement for determining the prestige or influence of an actor within its network. It can also be interpreted as how well connected a given actor is to other wellconnected actors. In this sense, prestige is a different measure of popularity; this difference comes from the fact that prestige considers the characteristics of the connections and if they are important themselves as well. The ten most influential nodes were risk factors (0.021), comorbidities (0.020), epidemiology $(0.020)$, treatment $(0.019)$, other cancer topography (0.019), chemoradiotherapy (0.019), post-treatment complications (0.019), molecular biology/biomarkers (0.019), potential malignant conditions (0.019), and screening (0.018). On the other hand, the constructs 
Table. Details of measured network vertices.

\begin{tabular}{|c|c|c|c|c|}
\hline Vertex & Degree & Betweenness Centrality & Closeness Centrality & Eigenvector Centrality \\
\hline access & 46 & 22.543 & 0.010 & 0.016 \\
\hline accuracy & 19 & 2.506 & 0.008 & 0.00607 \\
\hline adjunctive diagnostic aids & 32 & 8.620 & 0.008 & 0.011 \\
\hline africa & 9 & 0.00000 & 0.0071 & 0.00383 \\
\hline animal models & 27 & 2.139 & 0.008 & 0.010 \\
\hline antioxidants/chemoprevention & 47 & 15.931 & 0.010 & 0.016 \\
\hline asia & 40 & 5.981 & 0.009 & 0.015 \\
\hline behavior interventions & 30 & 3.663 & 0.008 & 0.011 \\
\hline biological process & 46 & 12.894 & 0.010 & 0.017 \\
\hline carcinogenesis & 49 & 27.593 & 0.010 & 0.017 \\
\hline chemo radiotherapy & 57 & 35.359 & 0.0108 & 0.019 \\
\hline clinical tissues & 46 & 11.301 & 0.010 & 0.017 \\
\hline comorbidities & 63 & 76.042 & 0.0115 & 0.0204 \\
\hline complementary tests & 37 & 6.459 & 0.009 & 0.014 \\
\hline cost effectiveness & 31 & 2.290 & 0.008 & 0.012 \\
\hline cytology & 26 & 2.621 & 0.008 & 0.00948 \\
\hline dental issues & 49 & 26.959 & 0.010 & 0.017 \\
\hline diagnosis/histopathology & 51 & 17.519 & 0.010 & 0.018 \\
\hline diagnostic delay & 21 & 3.791 & 0.008 & 0.00812 \\
\hline diet & 42 & 9.581 & 0.009 & 0.015 \\
\hline early detection & 47 & 29.364 & 0.010 & 0.015 \\
\hline environment & 26 & 1.944 & 0.008 & 0.010 \\
\hline epidemiology & 59 & 38.384 & 0.0110 & 0.0200 \\
\hline europe & 30 & 3.795 & 0.008 & 0.011 \\
\hline genomics & 49 & 21.047 & 0.010 & 0.017 \\
\hline head and neck cancer & 66 & 77.184 & 0.012 & 0.0214 \\
\hline health professionals & 35 & 17.382 & 0.009 & 0.011 \\
\hline help-seeking & 27 & 3.618 & 0.008 & 0.00945 \\
\hline HPV & 49 & 18.566 & 0.010 & 0.017 \\
\hline immunology & 36 & 2.849 & 0.009 & 0.014 \\
\hline inequalities & 32 & 9.861 & 0.008 & 0.011 \\
\hline knowledge & 29 & 7.026 & 0.008 & 0.010 \\
\hline laser & 22 & 3.454 & 0.008 & 0.00769 \\
\hline latin america & 14 & 0.14806 & 0.0074 & 0.00499 \\
\hline malignancies & 68 & 85.259 & 0.012 & 0.0217 \\
\hline medicine & 46 & 18.211 & 0.010 & 0.016 \\
\hline metastasis & 29 & 6.436 & 0.008 & 0.010 \\
\hline micro-organisms & 41 & 11.000 & 0.009 & 0.015 \\
\hline middle east & 14 & 0.38317 & 0.007 & 0.00532 \\
\hline
\end{tabular}


Innovative reflection on oral cancer research priorities: the contribution of social network analysis

\begin{tabular}{|c|c|c|c|c|}
\hline \multicolumn{5}{|l|}{ Continuation } \\
\hline molecular analysis & 37 & 11.287 & 0.009 & 0.013 \\
\hline molecular biology/biomarkers & 54 & 25.981 & 0.0104 & 0.019 \\
\hline mortality & 35 & 12.715 & 0.009 & 0.013 \\
\hline multidisciplinary care & 43 & 9.810 & 0.009 & 0.016 \\
\hline nanotechnology & 21 & 2.879 & 0.008 & 0.00730 \\
\hline north america & 12 & 0.06479 & 0.0074 & 0.00534 \\
\hline oceania & 7 & 0.13777 & 0.0070 & 0.00258 \\
\hline oral cancer & 73 & 114.213 & 0.013 & 0.0225 \\
\hline oral examination & 11 & 0.675 & 0.0072 & 0.00389 \\
\hline oral mucosal lesion & 28 & 3.462 & 0.008 & 0.010 \\
\hline oral rehabilitation & 15 & 0.09877 & 0.0074 & 0.00571 \\
\hline others cancer topography & 59 & 63.963 & 0.0110 & 0.019 \\
\hline palliative care & 21 & 0.505 & 0.008 & 0.00803 \\
\hline patient education & 38 & 11.338 & 0.009 & 0.013 \\
\hline policy & 49 & 16.975 & 0.010 & 0.017 \\
\hline post treatment complications & 57 & 40.298 & 0.0108 & 0.019 \\
\hline potential malignant conditions & 53 & 23.778 & 0.0103 & 0.019 \\
\hline prevention & 70 & 87.692 & 0.013 & 0.0221 \\
\hline professional education & 48 & 20.431 & 0.010 & 0.017 \\
\hline prognosis & 40 & 6.365 & 0.009 & 0.015 \\
\hline proms & 46 & 17.243 & 0.010 & 0.016 \\
\hline recurrence & 32 & 4.259 & 0.009 & 0.012 \\
\hline risk factors & 64 & 52.676 & 0.0116 & 0.0211 \\
\hline saliva & 24 & 0.937 & 0.008 & 0.00929 \\
\hline screening & 56 & 48.335 & 0.0106 & 0.018 \\
\hline self-examination & 13 & 0.26860 & 0.0074 & 0.00517 \\
\hline signs and symptoms & 38 & 7.878 & 0.009 & 0.014 \\
\hline staging & 37 & 14.983 & 0.009 & 0.014 \\
\hline study design & 69 & 85.872 & 0.012 & 0.0218 \\
\hline surgery & 44 & 11.357 & 0.009 & 0.016 \\
\hline survival & 47 & 14.459 & 0.010 & 0.017 \\
\hline technology & 9 & 0.19168 & 0.0072 & 0.00361 \\
\hline telemedicine & 10 & 0.49886 & 0.0072 & 0.00381 \\
\hline treatment & 57 & 26.627 & 0.0108 & 0.0198 \\
\hline vaccine & 48 & 24.040 & 0.010 & 0.017 \\
\hline
\end{tabular}

with less general centralities were Oceania, technology, telemedicine, Africa, oral examination, Latin America, self-examination, the Middle East, and North America.

The strongest relationships were between the nodes' antioxidants/chemoprevention and molecular biology/biomarkers, followed by biological processes and antioxidants/chemoprevention and antioxidants/ chemoprevention and oral cancer.

\section{Clusters}

Figure 3 shows a network that is grouped into clusters. Cluster 1 (dark blue) has 15 vertices and 35 


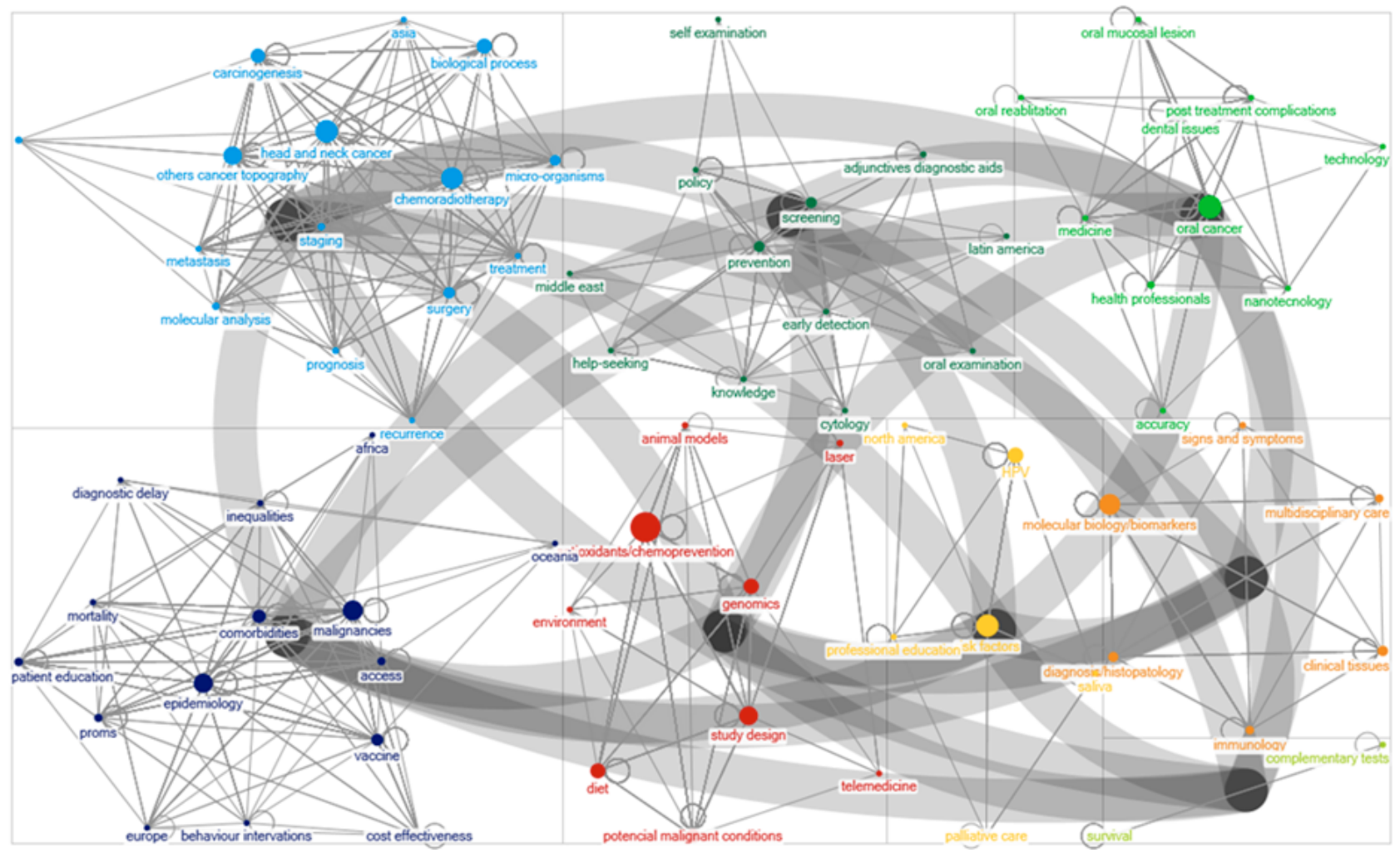

Figure 3. Clustered network graph

relationships between the nodes. Cluster 2 (blue) has 15 vertices and 23 relationships between nodes. Cluster 3 (dark green) has 12 vertices and adds 20 relationships between nodes. Cluster 4 (green) has 10 nodes and 12 unique edges. Cluster 5 (red) has nine vertices and ten unique edges. Cluster 6 (orange) and Cluster 7 (yellow) have six nodes and three unique edges. Finally, Cluster 8 (purple) has two nodes with one unique edge.

\section{Discussion}

The major goal of reducing oral cancer is to focus on preventive measures. Strategies for prevention have not been fully explored. ${ }^{2}$ To the best of our knowledge, this is the first network analysis to evaluate the interactions among keywords presented in articles published in the field of oral cancer prevention. Prevention is very important in the context of oral cancer, which still concentrates diagnosis in late stages, causing great morbidmortality. ${ }^{12}$ The main result of this analysis reveals key influential terms as risk factors, comorbidities, epidemiology, and treatment as the most influential nodes. However, it was interesting to note topics related to oral cancer prevention that remain foreign from central relations such as technology, telemedicine, oral examination, self-examination, and diagnostic delay are of value.

Although social network analysis differs from bibliometric studies, which use citation index to understand and present the volume of publications and their characteristics, the results point to the same path. Social network analysis uses centrality measures to indicate popularity and influence. In a bibliometric study published by Pena-Cristóbal et al., ${ }^{13}$ which analyzed the top 100 most-cited articles in oral cancer, the predominant area of study was the etiopathogenesis of oral cancer (42\%), followed by prognosis (16\%) and treatment (11\%), reinforcing the major connections within molecular research in the field of oral cancer. Along the same lines, Hassona et al. ${ }^{14}$ analyzed the 100 most-cited articles on squamous cell carcinoma of the mouth, lips, and 
oropharynx. Etiology and risk factors, especially $\mathrm{HPV}$, are the most commonly discussed topics. ${ }^{14}$

Telemedicine and technology were constructs that appeared as small centrality values. However, they may indicate a recent uniqueness, growing interest, and development. Telemedicine has the potential to promote equitable health care and streamline patient management, which could result in early detection of OC. ${ }^{15}$ It can improve the quality of primary healthcare by bridging the gap between primary and specialized healthcare. In view of the historical moment when the global population is experiencing the COVID19 pandemic, new technologies and prevention paths need to be discovered..$^{16}$ Hopefully, advances in telemedicine and new technologies will be seen in future studies.

The clustered network allowed us to understand some subgroups of relationships. The vertices in each cluster are not isolated from the relationships with other vertices belonging to other clusters. The thicker lines connecting the clusters in the chart demonstrate the relationships between them. It was not the purpose of this study to identify the place of origin of each publication geographically. However, where this data was available (indicated as a keyword) differences in oral cancer research could be noticed between the continents. It is interesting to note the relations of continents with different themes. Asia appears in the soft blue cluster, with constructs related to carcinogenesis, disease evolution, treatment, and prognosis. Asia has the highest rates of oral cancer worldwide due to cultural risk factors. ${ }^{17}$ The Middle East and Latin America are sub-grouped in the dark green cluster, with topics such as early detection and screening. These countries have a high prevalence of traditional risk factors related to tobacco use and strong social determinants, as in many emerging countries. ${ }^{18}$ North America appears closer to HPV and risk factors in the yellow cluster. Oceania, Africa, and Europe in the dark blue cluster are together with variables related to the patient (education, proms, and behavior interventions). A previous study warned of the notable lack of multicenter studies, indicating that there is a need to increase international collaboration in the field of oral cancer research. ${ }^{19}$
Cluster 1 (dark blue) included popular nodes, such as epidemiology and comorbidities. However, most variables related to patients, as education, behavior interventions, self-examination, and inequalities are also included in it and are clearly lacking in the literature. The subgroup is displaced when compared with another general view of the clustered network (Figure 2). It is well established that behavior is a risk factor in oral carcinogenesis, as a previous meta-analysis demonstrated that social determinants are an independent oral cancer risk factor. ${ }^{20}$ Therefore, it is important to advance patient awareness, as indicated by the high morbidity associated with oral cancer due to late diagnosis. Future studies should address this gap, as it could potentially be a low-cost and effective measure to improve prevention and early diagnosis.

Altimetric Explorer was used to analyze the top-100 articles on oral cancer that generated the highest online attention. It was found that treatment outcomes and quality of life were the most popular topics. ${ }^{14}$ Oral cancer can lead to serious and important social and effective consequences because the treatment may result in facial distortion, oral dysfunction, swallowing, and speech problems. ${ }^{21,22}$ Quality of life among oral cancer patients has become one of the most important parameters worth considering in diagnosis and post-treatment follow-up. Delayed diagnosis prevents successful treatment and favorable outcomes, resulting in the costliest and less effective treatment. ${ }^{23}$ In addition to the fact that diagnostic delay is presented as a small node, it is also isolated in the network. Since it has a great impact on prevention and treatment success, studies should be conducted to improve the diagnostic process.

Clusters 2 (blue) and 4 (green) concentrated on topics related to treatment and post-treatment complications. It is interesting to note that Cluster 3 (dark green), which presents betweenness centrality topics as early detection and screening, is in the middle of these two and can be seen as a connection point. In fact, high-risk target screening has demonstrated an impact on reducing mortality rates. ${ }^{24}$ Moreover, Cluster 5 (red) has central node oxidants and chemoprevention, including 
potential malignant conditions, diet, and genomics. Prevention through chemoprevention and/or the use of systemic medications is an extensively studied strategy and continues to hold promise as a way to diminish the negative indicators associated with oral cancer. ${ }^{25}$

Cluster 7 (yellow) draws attention to HPV, highlighting the fact that despite having a high degree or prevalence, it does not stand out in the network in relation to centrality values. HPV is the most commonly addressed topic and the most cited term among articles focused on etiology and risk factors. ${ }^{14}$ However, when analyzed in measures of centrality, one can notice that it does not occupy an articulated position in the network. Therefore, despite its great popularity, HPV has not built many interdisciplinary research bridges.

Cluster 6 (orange) points out that molecular biology/biomarkers are the most important nodes and appear close to signs and symptoms, diagnosis/ histopathology, and immunology. The strong relationship between this node and chemoprevention is due to biomarkers being expected to determine the effectiveness and safety of chemotherapy preventives. ${ }^{26}$ Finally, Cluster 8 (purple) resulted in only two nodes, survival and complementary tests, suggesting that tertiary prevention has not yet been articulated in the context of oral cancer prevention.

\section{Conclusion}

This study applied an innovative methodology to analyze published data in the field of oral cancer. However, there are some limitations to this study. The first is the omission of articles not detected by the search methods employed. The Scopus database is an important database and provides a good sample of present scientific production, but not all, and certainly, other important studies, might have been omitted. Second, the large number of keywords made the graphic interpretation more complex, which required additional categorization in constructs. Five specialists performed the review of the constructs; however, the results could not be easily replicated because of subjectivity in the interpretation of each term.

Nevertheless, within the limits of this methodology, it is possible to recognize publishing patterns in the field of oral cancer research and prevention. In contrast to bibliometric studies, network analysis enabled us to graphically visualize how different topics relate to recent research. It also facilitated recognition of the dominance and bias of biological and basic science in the field and identification of a need for studies concerning primary prevention, behavioral interventions, and inequalities in oral cancer. Future research should also investigate the gaps in network analysis and ways to encourage financial support and attention in these less-studied areas.

\section{References}

1. Maggioni D, Biffi L, Nicolini G, Garavello W. Flavonoids in oral cancer prevention and therapy. Eur J Cancer Prev. 2015 Nov;24(6):517-28. https://doi.org/10.1097/CEJ.0000000000000109

2. D'Souza S, Addepalli V. Preventive measures in oral cancer: an overview. Biomed Pharmacother. 2018 Nov; 107:72-80. https://doi.org/10.1016/j.biopha.2018.07.114

3. McLeod NM, Saeed NR, Ali EA. Oral cancer: delays in referral and diagnosis persist. Br Dent J. 2005 Jun;198(11):681-4. https://doi.org/10.1038/sj.bdj.4812381

4. Palmer O, Grannum R. Oral cancer detection [Internet]. Dent Clin North Am. 2011 Jul;55(3):537-48. https://doi.org/10.1016/i.cden.2011.02.009

5. Sankaranarayanan R, Ramadas K, Amarasinghe H, Subramanian S, Johnson N. Oral Cancer: Prevention, Early Detection, and Treatment. In: Gelband H, Jha P, Sankaranarayanan R, Horton S, editors. Cancer: disease control priorities. 3. ed. Washington, DC: World Bank Group; 2015. p. 85-99.

6. Hansen D, Shneiderman B, Smith MA. Analyzing social media networks with NodeXL: Insights from a connected world. Burlington: Morgan Kau; 2011.

7. Hansen DL. Exploring social media relationships. On the Horizon. 2011;19(1):43-51. https://doi.org/10.1108/10748121111107726.

8. Wasserman S, Faust K. Social network analysis: methods and applications. Cambridge; Cambridge University Press; 1994. 
- Innovative reflection on oral cancer research priorities: the contribution of social network analysis

9. Riddell J, Brown A, Kovic I, Jauregui J. Who are the most influential emergency physicians on twitter? West J Emerg Med. 2017 Feb;18(2):281-7. https://doi.org/10.5811/westjem.2016.11.31299

10. Liu GC, Odell JD, Whipple EC, Ralston R, Carroll AE, Downs SM. Data visualization for truth maintenance in clinical decision support systems. Int J Pediatr Adolesc Med. 2015 Jun;2(2):64-9. https://doi.org/10.1016/i.ijpam.2015.06.001

11. Uddin S, Mahmood H, Senarath U, Zahiruddin Q, Karn S, Rasheed S, et al. Analysis of stakeholders networks of infant and young child nutrition programmes in Sri Lanka, India, Nepal, Bangladesh and Pakistan. BMC Public Health. 2017 Jun;17(Suppl 2),405. https://doi.org/10.1186/s12889-017-4337-1

12. Güneri P, Epstein JB. Late stage diagnosis of oral cancer: components and possible solutions. Oral Oncol. 2014 Dec;50(12):1131-6. https://doi.org/10.1016/i.oraloncology.2014.09.005

13. Pena-Cristóbal M, Diniz-Freitas M, Monteiro L, Diz Dios P, Warnakulasuriya S. The 100 most cited articles on oral cancer. J Oral Pathol Med. 2018 Apr;47(4):333-44. https://doi.org/10.1111/jop.12686

14. Hassona Y, Qutachi T, Dardas L, Alrashdan MS, Sawair F. The online attention to oral cancer research: an Altmetric analysis. Oral Dis. 2019 Sep;25(6):1502-10. https://doi.org/10.1111/odi.13111

15. Carrard VC, Roxo Gonçalves M, Rodriguez Strey J, Pilz C, Martins M, Martins MD, et al. Telediagnosis of oral lesions in primary care: The EstomatoNet Program. Oral Dis. 2018 Sep;24(6):1012-9. https://doi.org/10.1111/odi.12851

16. Motta AC, Rodrigues KR H. Could we benefit from oral self-examination during the COVID-19 pandemic? Oral Oncol. 2020 Aug;17;104840. https://doi.org/10.1016/i.oraloncology.2020.104840

17. Sahu PK, Kumar S. Epidemiological aspects of oral cancer in North Indian population. Indian J Otolaryngol Head Neck Surg. 2019 Oct;71(S1 Suppl 1):944-8. https://doi.org/10.1007/s12070-019-01629-7

18. Johnson NW, Warnakulasuriya S, Gupta PC, Dimba E, Chindia M, Otoh EC, et al. Global oral health inequalities in incidence and outcomes for oral cancer: causes and solutions. Adv Dent Res. 2011 May;23(2):237-46. https://doi.org/10.1177/0022034511402082

19. Hassona Y, Qutachi T. A bibliometric analysis of the most cited articles about squamous cell carcinoma of the mouth, lips, and oropharynx. Oral Surg Oral Med Oral Pathol Oral Radiol. 2019 Jul;128(1):25-32e6. https://doi.org/10.1016/j.0000.2019.01.076

20. Conway DI, Petticrew M, Marlborough H, Berthiller J, Hashibe M, Macpherson LM. Socioeconomic inequalities and oral cancer risk: a systematic review and meta-analysis of case-control studies. Int J Cancer. 2008 Jun;122(12):2811-9. https://doi.org/10.1002/iic.23430

21. Warnakulasuriya S. Living with oral cancer: epidemiology with particular reference to prevalence and life-style changes that influence survival [Internet]. Oral Oncol. 2010 Jun;46(6):407-10. https://doi.org/10.1016/i.oraloncology.2010.02.015

22. Nieuwenhuizen AJ, Buffart LM, Brug J, Leemans CR, Verdonck-de Leeuw IM. The association between health related quality of life and survival in patients with head and neck cancer: a systematic review [Internet]. Oral Oncol. 2015 Jan;51(1):1-11. https://doi.org/10.1016/i.oraloncology.2014.09.002

23. More Y, D'Cruz AK. Oral cancer: review of current management strategies. Natl Med J India. 2013 May-Jun;26(3):152-8.

24. Sankaranarayanan R, Ramadas K, Thomas G, Muwonge R, Thara S, Mathew B, et al. Effect of screening on oral cancer mortality in Kerala, India: a cluster-randomised controlled trial. Lancet. 2005 Jun;365(9475):1927-33. https://doi.org/10.1016/S0140-6736(05)66658-5

25. Tanaka T, Tanaka M, Tanaka T. Oral carcinogenesis and oral cancer chemoprevention: a review. Patholog Res Int; 2011;2011:431246. https://doi.org/10.4061/2011/431246

26. Shah S, Kaur M. Biomarkers and chemopreventives in oral carcinogenesis and its prevention. J Oral Maxillofac Pathol. 2014 Jan;18(1):69-76. https://doi.org/10.4103/0973-029X.131914 